\title{
Burden of obesity in Mexico: prevalence, comorbidities, and associations with quality of life, resource utilisation and productivity
}

\author{
1. Marco DiBonaventura \\ Kantar Health, New York, NY, USA \\ 2. Agathe Le Lay \\ penhagen, Denmark \\ 3. Janine Fournier \\ Novo Nordisk, Copenhagen, Denmark \\ 4. Brandon Goode \\ Novo Nordisk Farmaceutica, São Paulo, Brazil \\ 5. Erik Bakker \\ Novo Nordisk, Mexico City, Mexico \\ Presenter: Rasmus Skovgaard \\ Novo Nordisk A/S
}

\section{Introduction}

- The prevalence of obesity in Mexico has risen substantially since the 1980s, with over 30\% of individuals in Mexico having obesity in 2010 . $^{1-2}$

- Obesity-related comorbidities such as cardiovascular disease and type 2 diabetes (T2D), ${ }^{3-4}$ have a profound economic impact on the Mexican medical system, totalling \$US 806 million in 2010

- However, no study has yet explored the relationship between obesity, Health-Related Quality of Life (HR-QoL) and work productivity.

\section{Objective}

mine the association between body mass index (BMI) and HR-QoL, work productivity, activity impairment healthcare resource utilisation (HCRU), and associated costs, in Mexico.

\section{Methods}

Sample and procedure

- Data were collected in 2014 using a cross-sectional survey of adults in Mexico $(\mathrm{N}=2511)$.

- Potential respondents were identified from an online pane with some offline recruitment to ensure representation in all demographic strata.

- The first 2011 respondents (the "general population") were recruited to reflect the demographics of Mexican adults based on the International Database of the United States Census. $^{5}$

- A supplemental 500 respondents with T2D were recruited to ensure adequate sample size for subgroup analyses.

\section{Measures}

- Demographics: age sex marital status, education employment, and socioeconomic status (SES).

- Health history: self-reported BMI class (underweight [BMI $\left.<18.5 \mathrm{~kg} / \mathrm{m}^{2}\right]$, normal weight [BMl 18.5- $\left.\leq 24.9 \mathrm{~kg} / \mathrm{m}^{2}\right]$ ), overweight [BMl $25-\leq 29.9 \mathrm{~kg} / \mathrm{m}^{2}$ ], obese class I [BM $30-\leq 34.9 \mathrm{~kg} / \mathrm{m}^{2}$, class II [BMI $35-\leq 39.9 \mathrm{~kg} / \mathrm{m}^{2}$ ] and class III $\left.\left[\mathrm{BMI} \geq 40 \mathrm{~kg} / \mathrm{m}^{2}\right]\right)$, smoking history, alcohol use and exercise frequency.

- Comorbidities: self-reported diagnosed comorbidities associated with the Charlson Comorbidity Index (CCI). Selfreported blood pressure (systolic; $>140 \mathrm{mmHg}$, diastolic; $>90 \mathrm{mmHg}$ ) was used to classify hypertension.

- Health outcomes: HR-QoL and the Work Productivity and Activity Impairment questionnaire were used to assess quality of life and overall work impairment. HCRU was self-reported.

Table 1 Regression-adjusted health outcome differences across BMI classes among the overall sample and those with T2D re-diabetes, and hypertension.

\begin{tabular}{|c|c|c|c|c|c|c|c|c|c|}
\hline \multirow[t]{2}{*}{$\begin{array}{l}\text { Dependent } \\
\text { variable }\end{array}$} & \multirow[t]{2}{*}{ BMI group $\left(\mathrm{kg} / \mathrm{m}^{2}\right)$} & \multicolumn{2}{|c|}{$\begin{array}{c}\text { Overall } \\
(\mathrm{N}=2476)\end{array}$} & \multicolumn{2}{|c|}{$\begin{array}{l}\text { Type } 2 \text { diabetes } \\
(\mathrm{N}=\mathbf{8 1 9})\end{array}$} & \multicolumn{2}{|c|}{$\begin{array}{l}\text { Pre-diabetes } \\
(\mathrm{N}=425)\end{array}$} & \multicolumn{2}{|c|}{$\begin{array}{l}\text { Hypertension } \\
(\mathrm{N}=\mathbf{8 2 5})\end{array}$} \\
\hline & & $\begin{array}{l}\text { Adjusted } \\
\text { mean }\end{array}$ & $p$-Value & $\begin{array}{l}\text { Adjusted } \\
\text { mean }\end{array}$ & $p$-Value & $\begin{array}{l}\text { Adjusted } \\
\text { mean }\end{array}$ & $p$-Value & $\begin{array}{l}\text { Adjusted } \\
\text { mean }\end{array}$ & $p$-Value \\
\hline $\begin{array}{l}\text { EQ-5D: Health } \\
\text { utility }\end{array}$ & $\begin{array}{l}\text { Overweight }(25 \text { to }<30) \\
\text { Obese class I }(30 \text { to }<35) \\
\text { Obese class II-III }(35+) \\
\text { Normal weight }(18.5 \text { to }<25)\end{array}$ & $\begin{array}{l}0.83 \\
0.82 \\
0.78 \\
0.85\end{array}$ & $\begin{array}{l}0.0187 \\
0.0003 \\
<.0001\end{array}$ & $\begin{array}{l}0.8 \\
0.77 \\
0.76 \\
0.81\end{array}$ & $\begin{array}{l}0.5873 \\
0.0172 \\
0.0043\end{array}$ & $\begin{array}{l}0.84 \\
0.83 \\
0.76 \\
0.85\end{array}$ & $\begin{array}{c}0.5398 \\
0.353 \\
0.0067\end{array}$ & $\begin{array}{l}0.79 \\
0.77 \\
0.74 \\
0.81\end{array}$ & $\begin{array}{c}0.1221 \\
0.0079 \\
<0.0001\end{array}$ \\
\hline $\begin{array}{l}\text { Overall work } \\
\text { impairment, \% }\end{array}$ & $\begin{array}{l}\text { Overweight }(25 \text { to }<30) \\
\text { Obese class I }(30 \text { to }<35) \\
\text { Obese class II-III }(35+) \\
\text { Normal weight }(18.5 \text { to }<25)\end{array}$ & $\begin{array}{l}20.34 \\
23.29 \\
25.81 \\
18.85\end{array}$ & $\begin{array}{l}0.2898 \\
0.0219 \\
0.0134\end{array}$ & $\begin{array}{l}26.62 \\
31.55 \\
30.49 \\
29.72\end{array}$ & $\begin{array}{l}0.2985 \\
0.6386 \\
0.8618\end{array}$ & $\begin{array}{l}18.48 \\
24.6 \\
40.14 \\
12.49\end{array}$ & $\begin{array}{l}0.2601 \\
0.0548 \\
0.0063\end{array}$ & $\begin{array}{l}27.17 \\
30.66 \\
31.4 \\
25.4\end{array}$ & $\begin{array}{l}0.5143 \\
0.0999 \\
0.1299\end{array}$ \\
\hline $\begin{array}{l}\text { Activity } \\
\text { impairment, \% }\end{array}$ & $\begin{array}{l}\text { Overweight }(25 \text { to }<30) \\
\text { Obese class I }(30 \text { to }<35) \\
\text { Obese class II-III }(35+) \\
\text { Normal weight }(18.5 \text { to }<25)\end{array}$ & $\begin{array}{l}18.31 \\
20.83 \\
24.82 \\
15.78\end{array}$ & $\begin{array}{c}0.038 \\
0.0023 \\
0.0003\end{array}$ & $\begin{array}{l}23.94 \\
28.88 \\
30.25 \\
23.55\end{array}$ & $\begin{array}{l}0.8742 \\
0.0982 \\
0.0793\end{array}$ & $\begin{array}{l}15.12 \\
15.84 \\
28.87 \\
11.69\end{array}$ & $\begin{array}{l}0.4741 \\
0.4129 \\
0.0411\end{array}$ & $\begin{array}{l}23.4 \\
28.3 \\
32.38 \\
21.99\end{array}$ & $\begin{array}{l}0.5419 \\
0.0247 \\
0.0047\end{array}$ \\
\hline $\begin{array}{l}\text { Number of } \\
\text { provider visits }\end{array}$ & $\begin{array}{l}\text { Overweight }(25 \text { to }<30) \\
\text { Obese class I }(30 \text { to }<35) \\
\text { Obese class II-III }(35+) \\
\text { Normal weight }(18.5 \text { to }<25)\end{array}$ & $\begin{array}{l}3.22 \\
3.4 \\
3.73 \\
3.09\end{array}$ & $\begin{array}{l}0.4151 \\
0.1319 \\
0.0235 \\
\end{array}$ & $\begin{array}{l}4.21 \\
4.87 \\
4.79 \\
4.74\end{array}$ & $\begin{array}{c}0.124 \\
0.7715 \\
0.9137 \\
.\end{array}$ & $\begin{array}{l}2.75 \\
2.84 \\
3.12 \\
2.59\end{array}$ & $\begin{array}{l}0.7135 \\
0.5945 \\
0.3667\end{array}$ & $\begin{array}{l}3.96 \\
4.13 \\
4.46 \\
4.13\end{array}$ & $\begin{array}{l}0.5938 \\
0.9957 \\
0.4361\end{array}$ \\
\hline Number of ER visits & $\begin{array}{l}\text { Overweight }(25 \text { to }<30) \\
\text { Obese class I }(30 \text { to }<35) \\
\text { Obese class II-III }(35+) \\
\text { Normal weight }(18.5 \text { to }<25)\end{array}$ & $\begin{array}{l}0.29 \\
0.35 \\
0.26 \\
0.24\end{array}$ & $\begin{array}{l}0.1414 \\
0.0126 \\
0.6042\end{array}$ & $\begin{array}{l}0.42 \\
0.72 \\
0.41 \\
0.43\end{array}$ & $\begin{array}{l}0.9373 \\
0.0271 \\
0.8506\end{array}$ & $\begin{array}{l}0.16 \\
0.23 \\
0.22 \\
0.1\end{array}$ & $\begin{array}{l}0.5282 \\
0.2616 \\
0.3459\end{array}$ & $\begin{array}{l}0.46 \\
0.58 \\
0.34 \\
0.34\end{array}$ & $\begin{array}{c}0.1387 \\
0.0161 \\
0.97\end{array}$ \\
\hline $\begin{array}{l}\text { Number of } \\
\text { hospitalisations }\end{array}$ & $\begin{array}{l}\text { Overweight }(25 \text { to }<30) \\
\text { Obese class I }(30 \text { to }<35) \\
\text { Obese class II-III }(35+) \\
\text { Normal weight }(18.5 \text { to }<25)\end{array}$ & $\begin{array}{l}0.17 \\
0.22 \\
0.22 \\
0.19\end{array}$ & $\begin{array}{l}0.3924 \\
0.4247 \\
0.6233\end{array}$ & $\begin{array}{l}0.27 \\
0.49 \\
0.31 \\
0.41\end{array}$ & $\begin{array}{l}0.0366 \\
0.3783 \\
0.3045\end{array}$ & $\begin{array}{l}0.11 \\
0.14 \\
0.19 \\
0.27\end{array}$ & $\begin{array}{c}0.2057 \\
0.328 \\
0.6293\end{array}$ & $\begin{array}{l}0.29 \\
0.36 \\
0.28 \\
0.31\end{array}$ & $\begin{array}{c}0.765 \\
0.5188 \\
0.7473\end{array}$ \\
\hline
\end{tabular}

Statistical analysis

- To obtain the prevalence of different glycaemic levels and cardiovascular comorbidities in the total adult Mexican population, weighting was applied to the prevalence within the general population sample.

- Comparisons between BMI categories were carried out using chi-squared tests and one-way ANOVAs.

- Generalised linear models were used to predict health outcomes, controlling for covariates.

- Analyses were replicated for those with T2D, pre diabetes, and hypertension.

- Indirect productivity costs were calculated by multiplying the mean level of productivity loss at each BMI class by the average wage in Mexico (12,850 MXN). ${ }^{6}$

\section{Results}

- The overall sample was $50.62 \%$ male with a mean age of $40.66( \pm 14.50)$ years and $40.62 \%$ belonging to the highest SES level ( $A B$; Higher \& intermediate managerial, administrative, professional occupations).

$1.39 \%$ were underweight, $35.88 \%$ were normal weight, $38.31 \%$ had overweight, $16.93 \%$ had class I obesity $5.26 \%$ class II, and $2.23 \%$ class III.

- Prevalence of glycaemic and cardiovascular comorbidities by BMl class is shown in Figure 1.

- Extrapolating the data to the total Mexican population estimated that 4.64 million adults currently have a BM $\geq 35 \mathrm{~kg} / \mathrm{m}^{2}$ and $75.2 \%$ are dysglycaemic

Figure 1 Prevalence of glycaemic levels and cardiovascula

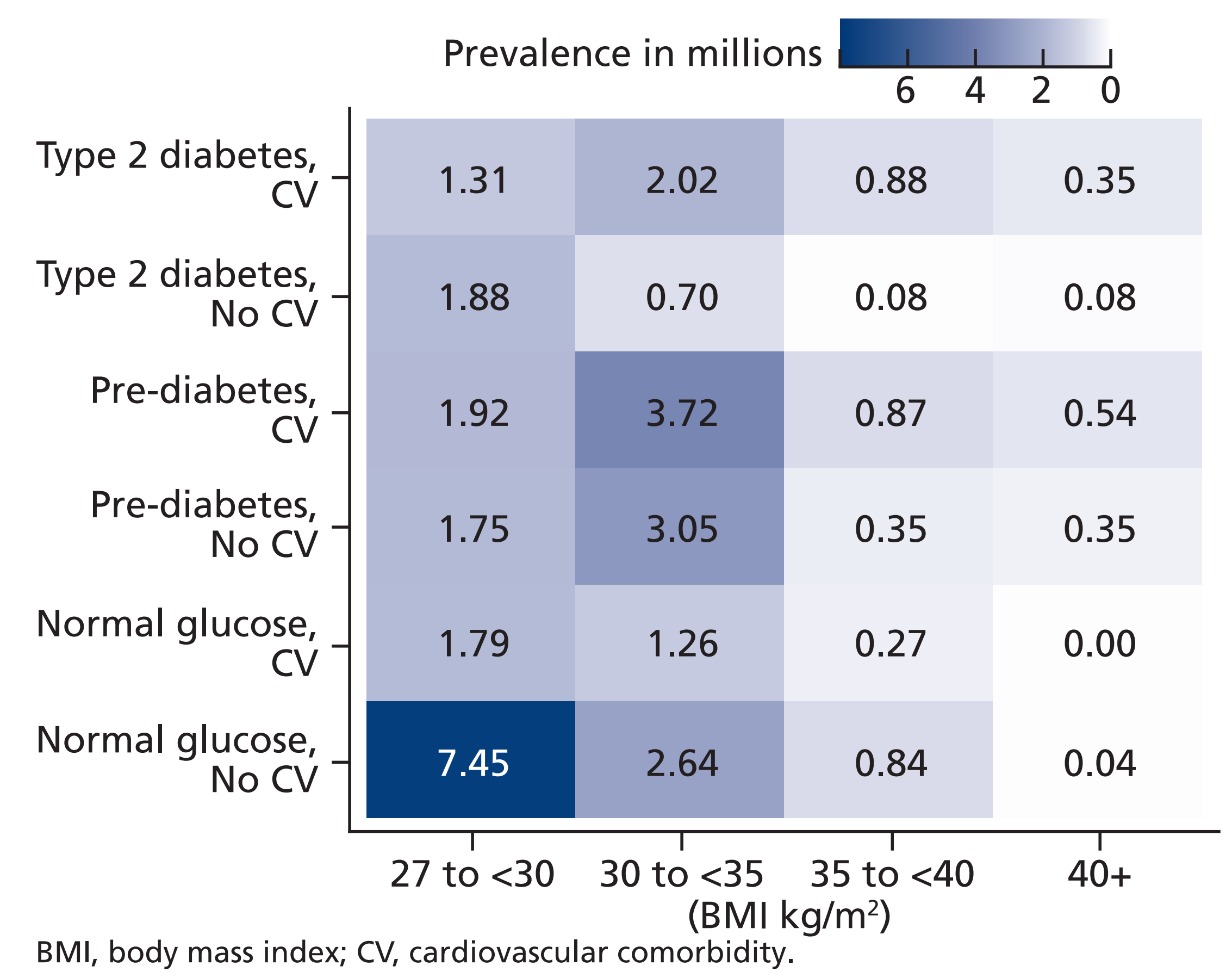

body mass index (BMI) class.

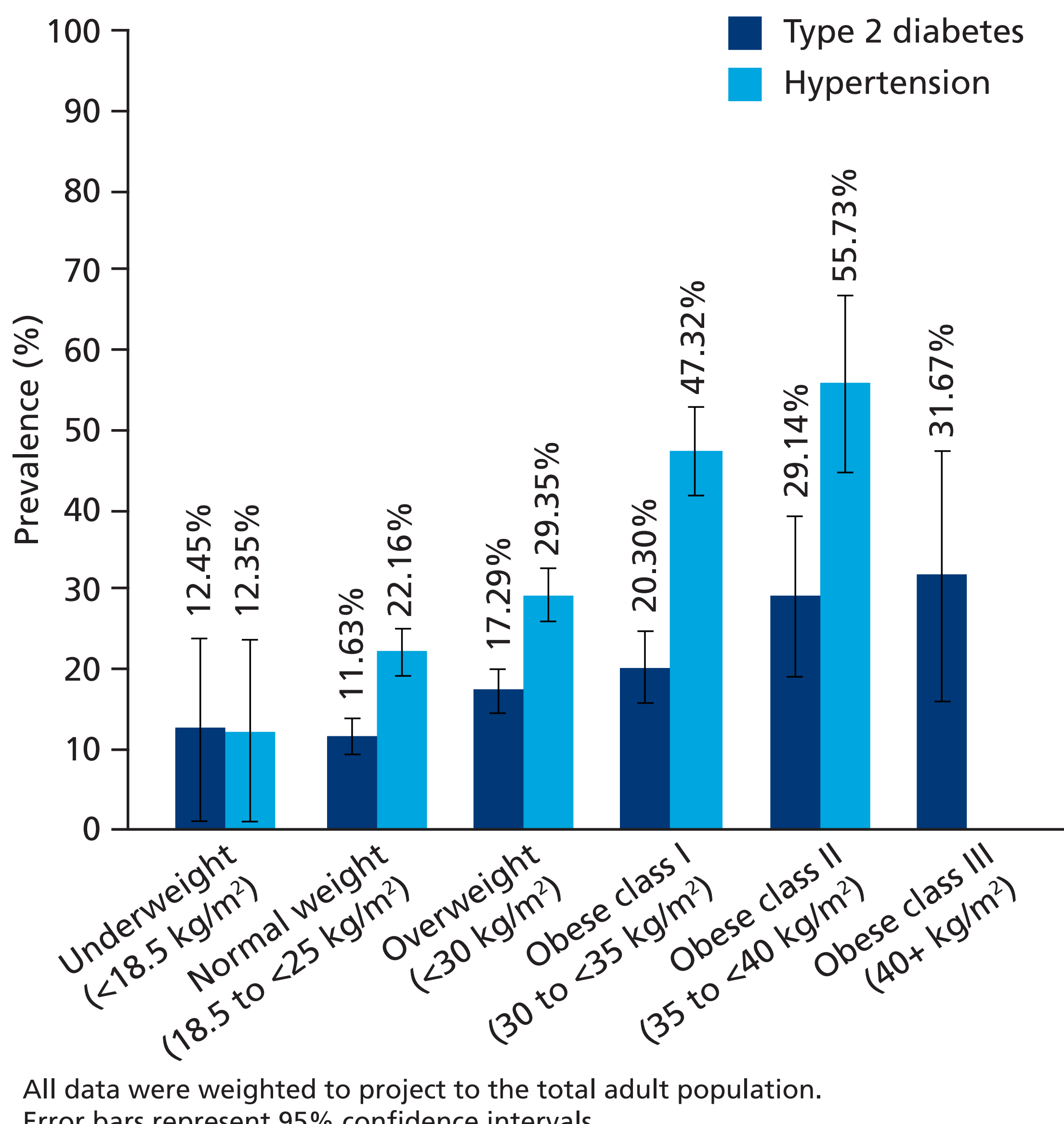

References

Soriano R et al. J Cancer Educ 2012;27:306-3

Rodriguez-Flores M et al. Cardiovasc Pathol 2013;22:332-8.

United States Census Bureau. International Database. Accessed August 23,

2017: http://www.census.gov/population/international/data/idb/

ormationGateway.php

OECD EConomic Surveys:

Conclusions

- The results suggest a significant effect of increasing BMI on comorbidities and a significant association between BMI class and health outcomes, particularly HR-QoL.

- Results were similar to the general population for patients with T2D, pre-diabetes, and hypertension. Given the severity of the obesity epidemic in Mexico, improvements in the epidemic in Mexico, improvements in the management of obesity could have significant benefits to the patient and larger society. 